\title{
Differential and partial cross sections of elastic and inelastic positronium-helium-atom scattering
}

\author{
Sadhan K. Adhikari \\ Instituto de Física Teórica, Universidade Estadual Paulista, 01.405-900 São Paulo, São Paulo, Brazil
}

(Received 28 April 2000; published 8 November 2000)

\begin{abstract}
Scattering of positronium (Ps) by a helium atom has been investigated in a three-Ps-state coupled-channel model including $\operatorname{Ps}(1 s, 2 s, 2 p)$ states using a recently proposed time-reversal-symmetric regularized electronexchange model potential. Specifically, we report results of differential cross sections for elastic scattering and target-elastic Ps excitations. We also present results for total and different partial cross sections and compare them with experiment and other calculations.
\end{abstract}

PACS number(s): $34.10 .+x, 36.10 . \mathrm{Dr}$

Scattering of the exotic orthopositronium atom with a long lifetime (142 ns) by neutral gas atoms and molecules is of fundamental interest in both physics and chemistry. Recent high-precision measurements of positronium (Ps) scattering by $\mathrm{H}_{2}, \mathrm{~N}_{2}, \mathrm{He}, \mathrm{Ne}, \mathrm{Ar}, \mathrm{C}_{4} \mathrm{H}_{10}$, and $\mathrm{C}_{5} \mathrm{H}_{12}$ [1-6] have enhanced theoretical activity [7-11] in this subject. Due to internal symmetry the direct static Born potential for elastic and even-parity transitions for these processes is zero and exchange correlation plays an important role in a correct description at low energies $[10,11]$.

Recently, we suggested [12] a regularized nonlocal electron-exchange model potential with a single parameter $C$ and used it in a successful study of Ps scattering by $\mathrm{H}$ $[13,14]$, $\mathrm{He}[12,15], \mathrm{Ne}[15], \mathrm{Ar}[15]$, and $\mathrm{H}_{2}$ [16,17]. Our results were in agreement with experimental total cross sections [1,3], especially at low energies for $\mathrm{He}, \mathrm{Ne}, \mathrm{Ar}$, and $\mathrm{H}_{2}$. In our initial calculations we used a nonsymmetric form of the model exchange potential for Ps scattering by $\mathrm{H}$ [14], He [12], and $\mathrm{H}_{2}$ [16]. Subsequent studies yielded improved results with a time-reversal-symmetric form of the model potential for Ps scattering by $\mathrm{H}[13]$ and $\mathrm{H}_{2}$ [17]. For $\mathrm{H}$ it was found [13] that the symmetric potential yielded excellent results for $S$-wave singlet Ps-H binding and resonance energies in agreement with accurate variational calculations [18]. The symmetric potential also led to very good results [15] for low-energy cross sections for Ps scattering by $\mathrm{He}, \mathrm{Ne}, \mathrm{Ar}$, and $\mathrm{H}_{2}$, in excellent agreement with experiment [3].

The problem of Ps-He scattering is of relevance to both experimentalists and theoreticians. Theoretically, it is the simplest of all Ps-scattering problems, and has reliable experimental cross sections. Once a good theoretical understanding of this system is obtained, we can try to understand the problem of Ps scattering by complex atoms and molecules. With this objective we reinvestigated the problem of Ps scattering by $\mathrm{He}$ at higher energies using the timereversal-symmetric form of the exchange potential. We consider a three-Ps-state coupled-channel model with $\operatorname{Ps}(1 s, 2 s, 2 p)$ states for calculating different elastic and inelastic cross sections of Ps-He scattering. We calculate the various Ps-He differential cross sections that are of great interest to experimentalists [5], in addition to the different angle-integrated partial cross sections. The differential cross sections carry detailed information about the scattering process. Cross sections for higher excitations and ionization of Ps are calculated by the Born approximation and added to the above $\operatorname{Ps}(1 s, 2 s, 2 p)$ cross sections to yield the total cross section, which is compared with experiment.

The theory for the coupled-channel study of Ps-He scattering with a regularized model potential has already appeared in the literature $[7,12,13,15]$. It is worthwhile to quote the relevant working equations here. For target-elastic Ps-He scattering we solve the following Lippmann-Schwinger scattering integral equation in momentum space for the total electronic doublet spin state:

$$
\begin{aligned}
f_{\nu^{\prime}, \nu}^{-}\left(\mathbf{k}^{\prime}, \mathbf{k}\right)= & \mathcal{B}_{\nu^{\prime}, \nu}^{-}\left(\mathbf{k}^{\prime}, \mathbf{k}\right) \\
& -\sum_{\nu^{\prime \prime}} \int \frac{d \mathbf{k}^{\prime \prime}}{2 \pi^{2}} \frac{\mathcal{B}_{\nu^{\prime}, \nu^{\prime \prime}}^{-}\left(\mathbf{k}^{\prime}, \mathbf{k}^{\prime \prime}\right) f_{\nu^{\prime \prime}, \nu}^{-}\left(\mathbf{k}^{\prime \prime}, \mathbf{k}\right)}{k_{\nu^{\prime \prime}}^{2} / 4-k^{\prime \prime 2} / 4+i 0}
\end{aligned}
$$

where the full Born amplitude $B^{-}$is given by $\mathcal{B}_{\nu^{\prime}, \nu}^{-}\left(\mathbf{k}^{\prime}, \mathbf{k}\right)$ $=g_{\nu^{\prime}, \nu}^{D}\left(\mathbf{k}^{\prime}, \mathbf{k}\right)-g_{\nu^{\prime}, \nu}^{E}\left(\mathbf{k}^{\prime}, \mathbf{k}\right)$, with $g^{D}$ and $g^{E}$ the direct and exchange Born amplitudes and $f^{-}$the scattering amplitude. The quantum states are labeled with the indices $\nu$ referring to the Ps atom. The variables $\mathbf{k}, \mathbf{k}^{\prime}, \mathbf{k}^{\prime \prime}$, etc., denote the appropriate momentum states; $\mathbf{k}_{\nu^{\prime \prime}}$ is the on-shell relative momentum of Ps with respect to $\mathrm{He}$ in channel $\nu^{\prime \prime}$. We use units $\hbar=m=1$ where $m$ is the electron mass. The differential cross section is given by

$$
\left(\frac{d \sigma}{d \Omega}\right)_{\nu^{\prime}, \nu}=\frac{k^{\prime}}{k}\left|f_{\nu^{\prime}, \nu}^{-}\left(\mathbf{k}^{\prime}, \mathbf{k}\right)\right|^{2} .
$$

For the He ground state, the space part of the HartreeFock (HF) wave function is given by $\Psi\left(\mathbf{r}_{1}, \mathbf{r}_{2}\right)$ $=\left[\varphi\left(\mathbf{r}_{1}\right) \varphi\left(\mathbf{r}_{2}\right)\right]$. The position vectors of the electrons are $\mathbf{r}_{1}$ and $\mathbf{r}_{2}$, and $\varphi$ is taken to be in the form $\varphi(\mathbf{r})=\Sigma_{\kappa} a_{\kappa} \phi_{\kappa}(\mathbf{r})$, where $\phi_{\kappa}(\mathbf{r})$ are the atomic orbitals.

The direct and exchange Born amplitudes are, respectively, given by $[12,15]$

$$
\begin{aligned}
g_{\nu^{\prime}, \nu}^{D}\left(\mathbf{k}_{f}, \mathbf{k}_{i}\right)= & \frac{4}{Q^{2}}\left[2-\sum_{\kappa, \kappa^{\prime}} a_{\kappa^{\prime}} a_{\kappa^{\prime}} \int \phi_{\kappa^{\prime}}^{*}(\mathbf{r})\right. \\
& \left.\times \exp (i \mathbf{Q} \cdot \mathbf{r}) \phi_{\kappa}(\mathbf{r}) d \mathbf{r}\right] \int \chi_{\nu^{\prime}}^{*}(\mathbf{t}) \\
& \times[2 i \sin (\mathbf{Q} \cdot \mathbf{t} / 2)] \chi_{\nu}(\mathbf{t}) d \mathbf{t}
\end{aligned}
$$


and

$$
\begin{aligned}
g_{\nu^{\prime}, \nu}^{E}\left(\mathbf{k}_{f}, \mathbf{k}_{i}\right)= & \sum_{\kappa, \kappa^{\prime}} \frac{4 a_{\kappa} a_{\kappa^{\prime}}(-1)^{l+l^{\prime}}}{D_{\kappa \kappa^{\prime}}} \int \phi_{\kappa^{\prime}}^{*}(\mathbf{r}) \\
& \times \exp (i \mathbf{Q} \cdot \mathbf{r}) \phi_{\kappa}(\mathbf{r}) d \mathbf{r} \int \chi_{\nu^{\prime}}^{*}(\mathbf{t}) \\
& \times \exp (i \mathbf{Q} \cdot \mathbf{t} / 2) \chi_{\nu}(\mathbf{t}) d \mathbf{t}
\end{aligned}
$$

with

$$
D_{\kappa, \kappa^{\prime}}=\left(k_{i}^{2}+k_{f}^{2}\right) / 8+C^{2}\left[\left(\alpha_{\kappa}^{2}+\alpha_{\kappa^{\prime}}^{2}\right) / 2+\left(\beta_{\nu}^{2}+\beta_{\nu^{\prime}}^{2}\right) / 2\right],
$$

where $l$ and $l^{\prime}$ are the angular momenta of the initial and final Ps states, the initial and final Ps momenta are $\mathbf{k}_{i}$ and $\mathbf{k}_{f}$, $\mathbf{Q}=\mathbf{k}_{i}-\mathbf{k}_{f}, \alpha_{\kappa}^{2} / 2$ and $\alpha_{\kappa^{\prime}}^{2}, 2$, and $\beta_{\nu}^{2}$ and $\beta_{\nu^{\prime}}^{2}$ are the binding energy parameters of the initial and final He orbital and Ps states in atomic units, respectively, and $C$ is the only parameter of the potential. Normally, the parameter $C$ is taken to be unity, which leads to reasonably good result $[15,17,23]$. However, it can be varied slightly from unity to get a precise fit to a low-energy observable. This variation of $C$ has no effect on the scattering observables at high energies where the model exchange potential reduces to the BornOppenheimer exchange potential [19]. In the present study we use the value $C=0.84$ throughout. This value of $C$ leads to a very good fit of the elastic Ps-He cross section with the experimental value of Skalsey et al. [3]. This exchange potential for Ps scattering is considered [12] to be a generalization of the Ochkur-Rudge exchange potential for electron scattering [20].

After a partial-wave projection, the system of coupled equations (1) is solved by the method of matrix inversion. A maximum number of partial waves $J_{\max }$ is included in solving the system of coupled equations. The differential and angle-integrated partial cross sections so calculated are augmented by Born results for higher partial waves $J>J_{\max }$. A maximum of 40 Gauss-Legendre quadrature points is used in the discretization of each momentum-space integral. The calculations are performed with the exact Ps wave functions and the HF orbitals for the He ground state [21]. Although it is relatively easy to obtain converged results for angleintegrated partial cross sections, special care is needed to obtain converged results for differential cross sections at higher energies. Coverged results for partial cross sections are obtained for $J_{\max }=30$ at all energies. To obtain convergent differential cross sections, we need to take $J_{\max }=150$ partial waves at $100 \mathrm{eV}$. However, $J_{\max }=30$ is sufficient for obtaining convergent differential cross sections at 20 and 30 eV.

Here we present results of Ps-He scattering using a threePs-state model that includes the following states: $\mathrm{Ps}(1 s) \mathrm{He}(1 s 1 s), \mathrm{Ps}(2 s) \mathrm{He}(1 s 1 s)$, and $\mathrm{Ps}(2 p) \mathrm{He}(1 s 1 s)$. The Born terms for the excitation of $\mathrm{He}$ are found to be small and are not considered here in the coupled-channel scheme. First, we present the elastic $\operatorname{Ps}(1 s) \mathrm{He}(1 s 1 s)$ differential

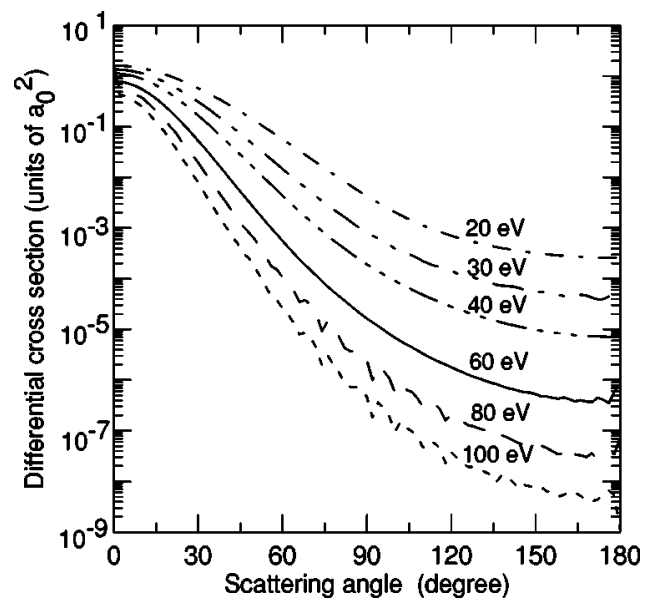

FIG. 1. Differential cross section (in units of $a_{0}^{2}$ ) for elastic Ps-He scattering at the following incident Ps energies: $20 \mathrm{eV}$ (dashed-dotted line), $30 \mathrm{eV}$ (dashed-double-dotted line), $40 \mathrm{eV}$ (dashed-triple-dotted line), $60 \mathrm{eV}$ (full line), 80 (long dashed line), and $100 \mathrm{eV}$ (short dashed line).

cross section and inelastic differential cross sections for scattering to $\mathrm{Ps}(2 s) \mathrm{He}(1 s 1 s)$ and $\mathrm{Ps}(2 p) \mathrm{He}(1 s 1 s)$ states at different energies.

In order to show the general trend of the differential cross sections, we perform calculations at the following incident positronium energies: 20, 30, 40, 60, 80, and $100 \mathrm{eV}$. We exhibit the differential cross sections for elastic scattering at these energies in Fig. 1. In Figs. 2 and 3 we show the inelastic cross sections for transition to $\mathrm{Ps}(2 s) \mathrm{He}(1 s 1 s)$ and $\mathrm{Ps}(2 p) \mathrm{He}(1 s 1 s)$ states. From all these figures we find that, as expected, the differential cross sections are more isotropic at low energies where only the low partial waves contribute. At higher energies more and more partial waves are needed to achieve convergence and the differential cross sections are more anisotropic. The small oscillation of the differential cross sections at larger angles and energies is due to numerical difficulties.

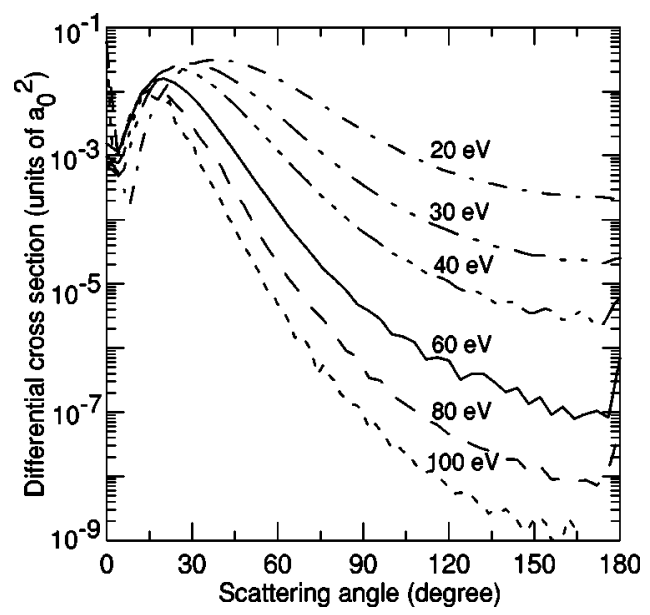

FIG. 2. Differential cross section (in units of $a_{0}^{2}$ ) for inelastic Ps-He scattering to $\mathrm{Ps}(2 s) \mathrm{He}(1 s 1 s)$ state at the following incident Ps energies: $20 \mathrm{eV}$ (dashed-dotted line), $30 \mathrm{eV}$ (dashed-doubledotted line), $40 \mathrm{eV}$ (dashed-triple-dotted line), $60 \mathrm{eV}$ (full line), 80 (long dashed line), and $100 \mathrm{eV}$ (short dashed line). 


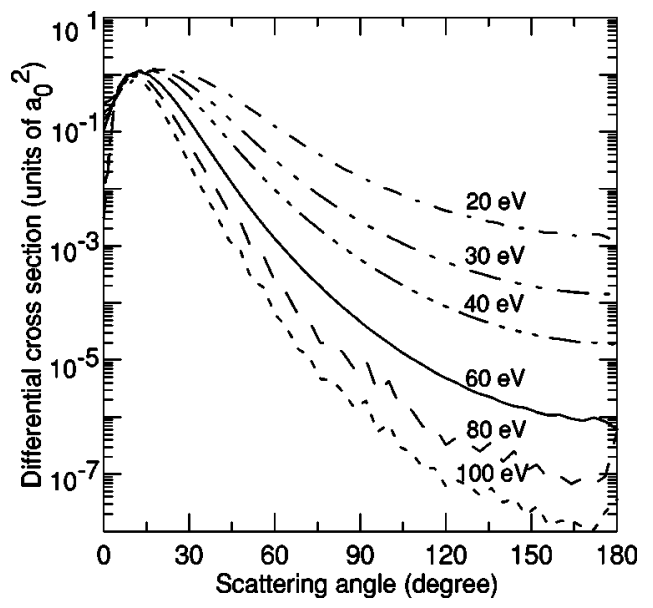

FIG. 3. Differential cross section (in units of $a_{0}^{2}$ ) for inelastic Ps-He scattering to $\mathrm{Ps}(2 p) \mathrm{He}(1 s 1 s)$ state at the following incident Ps energies: $20 \mathrm{eV}$ (dashed-dotted line), $30 \mathrm{eV}$ (dashed-doubledotted line), $40 \mathrm{eV}$ (dashed-triple-dotted line), $60 \mathrm{eV}$ (full line), 80 (long dashed line), and $100 \mathrm{eV}$ (short dashed line).

Recently, Garner et al. [5] have provided an experimental estimate of average differential cross sections across the energy range 10 to $100 \mathrm{eV}$ with respect to any process in Ps-He scattering for forward scattering angles: $\langle d \sigma / d \Omega\rangle=(34$ $\pm 12) \times 10^{-20} \mathrm{~m}^{2} \mathrm{sr}^{-1}=(121 \pm 43) a_{0}^{2} \mathrm{sr}^{-1}$. However, it is not possible to make a meaningful comparison between the present differential cross sections and the experimental estimate of Garner et al.

We calculate the different angle-integrated partial cross sections for Ps-He scattering. In addition to the $\mathrm{Ps}(1 s, 2 s, 2 p)$ cross sections calculated using the coupled-channel method, we also calculate the higher Ps $(7>n>2)$ excitation and Ps ionization cross sections using the Born approximation with the present exchange potential. These results are shown in Fig. 4, where we plot angle-integrated elastic, Ps $(n=2)$

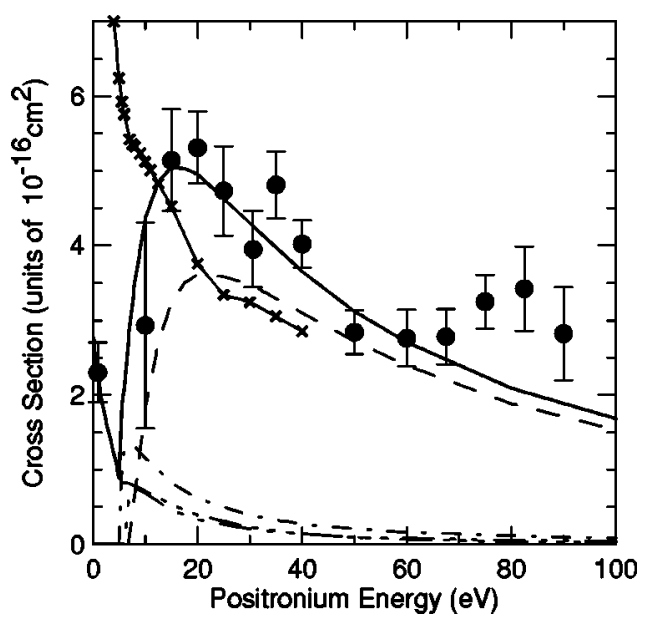

FIG. 4. Partial and total cross sections (in units of $10^{-16} \mathrm{~cm}^{2}$ ) of Ps-He scattering at different Ps energies: Ps $(1 s)$ (dashed-tripledotted line), Ps $(n=2)$ (dashed-dotted line), Ps $(7>n>2)$ (dasheddouble-dotted line), Ps ionization (dashed line), total (full line), total from Ref. [8] (full line with crosses), and data points with error bars from Refs. [1,3]. $[\equiv \operatorname{Ps}(2 s+2 p)]$, inelastic Ps $(7>n>2)$, and Ps ionization cross sections. The total cross section calculated from these partial cross sections is also shown in this plot and compared with the experiments of Refs. [1,3] and the total cross section of the 22-Ps-state $R$-matrix calculation of Ref. [8]. The agreement between theory and experiment is quite good up to $70 \mathrm{eV}$. The target-inelastic processes ignored in this work are supposed to play an important role at higher energies, which may be why the agreement of the present results with experiment deteriorates above $70 \mathrm{eV}$. There exists qualitative disagreement between the present total cross section and that of the 22-state calculation of Ref. [8], on which we comment below.

As the Ps-He system is of fundamental interest to both theoreticians and experimentalists, it is appropriate to compare our results critically with those of other theories and experiments. The only other recent experiment on Ps-He is the one by Nagashima et al. [4], who obtained the cross sec-

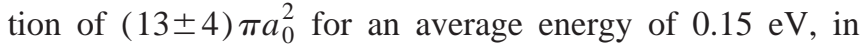
striking disagreement with the present calculation yielding $2.58 \pi a_{0}^{2}$ at $0.9 \mathrm{eV}$ as well as with the experiment of Skalsey et al. [3] who obtained (2.61 \pm 0.5$) \pi a_{0}^{2}$ at about $0.9 \mathrm{eV}$.

The independent experiment on measurement [22] of the pick-off quenching rate of Ps on $\mathrm{He}$ can be used [23] to resolve the stalemate. It is argued [23] that a large lowenergy Ps-He elastic cross section implies a large repulsive exchange potential between Ps and He atoms in the elastic channel. In the presence of a large repulsive potential it will be difficult for the Ps atom to approach the He atom. Consequently, one will have a small value for the pick-off quenching rate. From a study of the pick-off quenching rates of different models, we concluded [23] that a small lowenergy cross section, as obtained by us, will lead to a large pick-off quenching rate in agreement with experiment. The large low-energy cross sections obtained in other theoretical models $[7,8,10,11]$ will lead to a much too small pick-off quenching rate in disagreement with experiment. The present low-energy cross section and the experiment of Skalsey et al. [3] are consistent with the pick-off quenching rate measurement [22]. It would be difficult to reconcile the low-energy cross section of Nagashima et al. [4] and other theoretical results $[7,8,10,11]$ with the measurement of the pick-off quenching rate.

We note that a model calculation by Peach [24], performed before the experiment of Skalsey et al. [3], is also in reasonable agreement with the present calculation and lowenergy experiments. The model of Peach was constructed by fitting to known positron-helium [25] and electron-helium [26] scattering data.

In Table I we compare the results for the angle-integrated partial cross sections to $\operatorname{Ps}(1 s, 2 s, 2 p)$ states of different theoretical calculations. The present $\operatorname{Ps}(1 s)$ Born cross sections are much smaller than the Born-Oppenheimer cross sections [19] used as input to close-coupling [7] or $R$-matrix [8] schemes. There have been different static-exchange calculations on Ps-He since the 1960s $[7,8,10,11]$. These calculations yielded similar results and in the static-exchange (SE) column of Table I we quote the recent cross sections of Refs. 
TABLE I. Angle-integrated Ps-He partial cross sections in units of $\pi a_{0}^{2}$ at different positronium energies. $\mathrm{EB}$, first Born approximation with present exchange; BO, first Born approximation with Born-Oppenheimer exchange [7]; SE, static exchange of Refs. [7,8]; 3St, three-Ps-state with present exchange; 22St, 22-Ps-state $R$-matrix calculation of Ref. [8].

\begin{tabular}{|c|c|c|c|c|c|c|c|c|c|c|c|}
\hline \multirow{2}{*}{$\begin{array}{l}\text { Energy } \\
(\mathrm{eV})\end{array}$} & \multicolumn{3}{|c|}{ EB } & \multirow{2}{*}{$\begin{array}{c}\mathrm{BO} \\
\operatorname{Ps}(1 s)\end{array}$} & \multirow{2}{*}{$\begin{array}{c}\mathrm{SE} \\
\operatorname{Ps}(1 s)\end{array}$} & \multirow{2}{*}{$\begin{array}{c}22 \mathrm{St} \\
\operatorname{Ps}(1 s)\end{array}$} & \multicolumn{4}{|c|}{$3 \mathrm{St}$} & \multirow{2}{*}{$\begin{array}{l}22 \mathrm{St} \\
\mathrm{Ps}(2)\end{array}$} \\
\hline & $\operatorname{Ps}(1 s)$ & $\operatorname{Ps}(2 s)$ & $\operatorname{Ps}(2 p)$ & & & & $\operatorname{Ps}(1 s)$ & $\operatorname{Ps}(2 s)$ & $\operatorname{Ps}(2 p)$ & $\operatorname{Ps}(2)$ & \\
\hline 0 & 15.82 & & & & 14.6 & 13.2 & 3.34 & & & & \\
\hline 0.068 & 15.33 & & & 132 & 14.4 & 13.0 & 3.15 & & & & \\
\hline 0.612 & 12.11 & & & 98 & 12.9 & & 2.75 & & & & \\
\hline 1.088 & 10.04 & & & 78 & 12.1 & 11.3 & 2.48 & & & & \\
\hline 1.7 & 8.08 & & & 59 & 11.3 & & 2.18 & & & & \\
\hline 2.448 & 6.38 & & & 44 & 10.5 & 9.4 & 1.88 & & & & \\
\hline 4.352 & 3.91 & & & 23 & 9.0 & & 1.26 & & & & \\
\hline 5 & 3.39 & & & & 8.6 & 7.1 & 1.00 & & & & \\
\hline 5.508 & 3.06 & 0.070 & 1.44 & & & & 0.96 & 0.071 & 1.15 & 1.22 & 0.24 \\
\hline 6 & 2.79 & 0.091 & 1.78 & & 8.1 & 6.1 & 0.97 & 0.083 & 1.35 & 1.43 & 0.42 \\
\hline 6.8 & 2.42 & 0.100 & 1.89 & 12 & 7.7 & & 0.96 & 0.074 & 1.47 & 1.54 & \\
\hline 8 & 1.99 & 0.097 & 1.77 & & 7.1 & 4.8 & 0.92 & 0.056 & 1.45 & 1.51 & 0.50 \\
\hline 10 & 1.51 & 0.080 & 1.48 & & 6.7 & 3.8 & 0.84 & 0.048 & 1.29 & 1.34 & 0.51 \\
\hline 15 & 0.86 & 0.048 & 0.97 & 3.0 & 4.8 & 2.4 & 0.63 & 0.042 & 0.91 & 0.95 & 0.44 \\
\hline 20 & 0.56 & 0.031 & 0.70 & 1.7 & 3.6 & 1.5 & 0.46 & 0.032 & 0.67 & 0.70 & 0.31 \\
\hline 30 & 0.29 & 0.016 & 0.43 & 0.6 & 2.0 & 1.0 & 0.27 & 0.017 & 0.42 & 0.44 & 0.18 \\
\hline 40 & 0.17 & 0.0094 & 0.30 & 0.22 & 0.7 & 0.8 & 0.17 & 0.010 & 0.30 & 0.31 & 0.12 \\
\hline 50 & 0.11 & 0.0061 & 0.23 & & & & 0.11 & 0.0067 & 0.23 & 0.24 & \\
\hline 60 & 0.079 & 0.0042 & 0.18 & 0.04 & 0.08 & & 0.077 & 0.0045 & 0.18 & 0.19 & \\
\hline 80 & 0.043 & 0.0023 & 0.13 & 0.007 & 0.01 & & 0.042 & 0.0024 & 0.13 & 0.13 & \\
\hline 100 & 0.026 & 0.0014 & 0.10 & 0.001 & 0.002 & & 0.026 & 0.0014 & 0.10 & 0.10 & \\
\hline 150 & 0.010 & 0.0005 & 0.06 & & & & 0.010 & 0.0005 & 0.06 & 0.06 & \\
\hline
\end{tabular}

[7,8]. Although these SE cross sections are much smaller than the corresponding Born-Oppenheimer cross sections, they are much larger than those of the present calculation. The 22-Ps-state $R$-matrix calculation [8] yields elastic cross sections marginally smaller than the SE cross sections, and it seems unlikely that a "converged" $R$-matrix calculation will lead to elastic cross sections comparable to the present ones. However, the measured pick-off quenching rate [22] favors [23] a weak exchange potential and small Ps $(1 s)$ cross sections at low energies, and future measurements of lowenergy Ps-He elastic cross sections will decide which of the results are more realistic. Although the present elastic Ps(1) cross sections are much smaller than those of the $R$-matrix calculation, the reverse is true for the excitation cross sections to the Ps(2) states as can be seen in Table I. The large Ps excitation (and Ps ionization) cross sections of the present calculation and the small low-energy elastic cross sections are collectively responsible for the construction of the pronounced peak in the total cross section shown in Fig. 4 near $15-20 \mathrm{eV}$, in agreement with the experiments of Refs. [1] and [3]. This peak is also present in the calculation of Peach [24] and is clearly absent in the close-coupling [7] and 22Ps-state $R$-matrix analyses [8]. Similar peaks also appear in the total cross sections of Ps- $\mathrm{H}_{2}$ and Ps-Ar scattering [5].

To summarize, we have performed a three-Ps-state coupled-channel calculation of Ps-He scattering at low and medium energies using a regularized symmetric nonlocal electron-exchange model potential recently suggested by us and successfully used in other Ps scattering problems. We present results for differential cross sections at several incident Ps energies between $20 \mathrm{eV}$ and $100 \mathrm{eV}$ for elastic scattering and inelastic excitation to $\mathrm{Ps}(2 s, 2 p) \mathrm{He}(1 s 1 s)$ states. We also present the angle-integrated partial cross sections and compare them with those of other calculations. The present total cross sections are in agreement with data of Refs. $[1,3]$. However, there is alarming discrepancy between the present cross sections and those of conventional $R$-matrix [8] and close-coupling [7] calculations. These latter calculations are in agreement with a recent measurement of lowenergy cross sections by Nagashima et al. [4]. At low energies, the present elastic cross sections are much too small compared to those of Refs. $[7,8]$. However, the present total cross section develops a pronounced maximum near 15-20 $\mathrm{eV}$ as can be seen in Fig. 4 in agreement with the general experimental trend [5]. The cross section of Ref. [8] does not have this behavior. Although comparison with pick-off quenching measurement data [22] at low-energy favors [23] the results of the present model, further precise measurements of total and Ps(2) excitations at low energies will finally resolve the stalemate.

The work was supported in part by the Conselho Nacional de Desenvolvimento-Científico e Tecnológico, Fundação de Amparo à Pesquisa do Estado de São Paulo, and Financiadora de Estudos e Projetos of Brazil. 
[1] A. J. Garner, G. Laricchia, and A. Özen, J. Phys. B 29, 5961 (1996).

[2] N. Zafar, G. Laricchia, M. Charlton, and A. Garner, Phys. Rev. Lett. 76, 1595 (1996); A. J. Garner and G. Laricchia, Can. J. Phys. 74, 518 (1996); A. J. Garner, A. Özen, and G. Laricchia, Nucl. Instrum. Methods Phys. Res. B 143, 155 (1998).

[3] M. Skalsey, J. J. Engbrecht, R. K. Bithell, R. S. Vallery, and D. W. Gidley, Phys. Rev. Lett. 80, 3727 (1998).

[4] Y. Nagashima, T. Hyodo, K. Fujiwara, and A. Ichimura, J. Phys. B 31, 329 (1998).

[5] A. J. Garner, A. Özen, and G. Laricchia, J. Phys. B 33, 1149 (2000).

[6] H. H. Andersen, E. A. G. Armour, J. W. Humberston, and G. Laricchia, Nucl. Instrum. Methods Phys. Res. B 143, U10 (1998).

[7] N. K. Sarkar and A. S. Ghosh, J. Phys. B 30, 4591 (1997); N. K. Sarka, P. Chaudhury, and A. S. Ghosh, ibid. 32, 1657 (1999).

[8] J. E. Blackwood, C. P. Campbell, M. T. McAlinden, and H. R. J. Walters, Phys. Rev. A 60, 4454 (1999).

[9] H. Ray, J. Phys. B 32, 5681 (1999); 33, 4285 (2000); Phys. Lett. A 252, 316 (1999).

[10] M. I. Barker and B. H. Bransden, J. Phys. B 1, 1109 (1968); 2, 730 (1969).

[11] P. A. Fraser, J. Phys. B 1, 1006 (1968); P. A. Fraser and M. Kraidy, Proc. Phys. Soc. London 89, 553 (1966).

[12] P. K. Biswas and S. K. Adhikari, Phys. Rev. A 59, 363 (1999).
[13] S. K. Adhikari and P. K. Biswas, Phys. Rev. A 59, 2058 (1999).

[14] P. K. Biswas and S. K. Adhikari, J. Phys. B 31, 3147 (1998).

[15] P. K. Biswas and S. K. Adhikari, Chem. Phys. Lett. 317, 129 (2000); P. K. Biswas, Radiat. Phys. Chem. 58, 443 (2000); Phys. Rev. A 61, 012502 (2000).

[16] P. K. Biswas and S. K. Adhikari, J. Phys. B 31, L737 (1998); 31, L315 (1998).

[17] P. K. Biswas and S. K. Adhikari, J. Phys. B 33, 1575 (2000).

[18] Z. C. Yan and Y. K. Ho, Phys. Rev. A 59, 2697 (1999); 60, 5098 (1999); A. M. Frolov and V. H. Smith, Jr., ibid. 55, 2662 (1997); N. Jiang and D. M. Schrader, Mater. Sci. Forum 255-2, 312 (1997).

[19] J. R. Oppenheimer, Phys. Rev. 32, 361 (1928); S. K. Adhikari and P. Mandal, J. Phys. B 33, L761 (2000).

[20] M. H. R. Rudge, Proc. Phys. Soc. London 86, 763 (1965); V. I. Ochkur, Zh. Éksp. Teor. Fiz. 45, 734 (1963) [Sov. Phys. JETP 18, 503 (1964)].

[21] E. Clementi and C. Roetti, At. Data Nucl. Data Tables 14, 177 (1974).

[22] B. G. Duff and F. F. Heymann, Proc. R. Soc. London, Ser. A 270, 517 (1962); F. F. Heymann, P. E. Osmon, J. J. Veit, and W. F. Williams, Proc. Phys. Soc. London 78, 1038 (1961).

[23] S. K. Adhikari, P. K. Biswas, and R. A. Sultanov, Phys. Rev. A 59, 4829 (1999).

[24] G. Peach (unpublished) quoted in Refs. [1,8].

[25] J. W. Humberston and R. I. Campeanu, J. Phys. B 13, 4907 (1980).

[26] R. K. Nesbet, Phys. Rev. A 20, 58 (1979). 